\title{
Omnipresent coexistence of rogue waves in a nonlinear two-wave interference system and its explanation by modulation instability
}

\author{
Changchang Pan, ${ }^{1}$ Lili Bu, ${ }^{1}$ Shihua Chen $\odot,{ }^{1,{ }^{*}}$ Dumitru Mihalache $\odot,{ }^{2}$ Philippe Grelu $\odot,{ }^{3}$ and Fabio Baronio ${ }^{4, \dagger}$ \\ ${ }^{1}$ School of Physics and Quantum Information Research Center, Southeast University, Nanjing 211189, China \\ ${ }^{2}$ Department of Theoretical Physics, Horia Hulubei National Institute for Physics and Nuclear Engineering, \\ Magurele-Bucharest, RO-077125, Romania \\ ${ }^{3}$ Laboratoire ICB, U.M.R. 6303 C.N.R.S., Université Bourgogne Franche-Comté, 9 avenue A. Savary, F-21078 Dijon, France \\ ${ }^{4}$ INO CNR and Dipartimento di Ingegneria dell'Informazione, Università di Brescia, Via Branze 38, 25123 Brescia, Italy
}

(Received 9 May 2021; accepted 2 August 2021; published 13 August 2021)

\begin{abstract}
We investigate the coexisting rogue wave dynamics associated with two fundamental-frequency optical waves interacting in a quadratic nonlinear medium. Using the vector Chen-Lee-Liu nonlinear Schrödinger equation model, we obtain exact rogue wave solutions at first and higher orders on the more general periodic backgrounds. We unveil that the inherent self-steepening effect may allow an omnipresent rogue wave coexistence over a broad range of parameters in both the normal and anomalous dispersion regimes, in addition to allowing ultrastrong peak amplitudes. We also demonstrate that such universality of coexistence can be anticipated by the appearance of two peaks in the modulation instability spectrum. We numerically confirm the robustness of the coexisting Peregrine solitons against initial noise as well as their excitation from a turbulent wave field caused by modulation instability. We expect that these findings will shed light on the generation of extreme wave events resulting from the interference of multiple continuous-wave fields.
\end{abstract}

DOI: 10.1103/PhysRevResearch.3.033152

\section{INTRODUCTION}

The study of deterministic rogue wave events of integrable models [1-3] has attracted an increasing interest in the past decade, in diverse disciplines ranging from hydrodynamics [4,5] to optics and photonics [6-8], acoustics [9], magnetics [10], Bose-Einstein condensation [11,12], and even artificial intelligence [13]. Theoretical investigations usually involve a complicated construct based on the integrability of a class of nonlinear partial differential equations [14], by which researchers can gain a microscopic insight into the mysterious rogue wave phenomena seen in the real world [15-18]. Particularly, this has enabled oceanographers to explore, in a safe way, the infamous oceanic rogue waves, which cast a devastating power and seem to be appearing out of nowhere [19]. In the optical context, understanding the determinism of such events helps experimentalists to prepare the delicate set of parameters and initial conditions leading to the effective observation of rogue waves [7,20-22].

The well-known Peregrine soliton is a central figure of deterministic rogue waves [23]. It is a transient wave packet localized in space and time with a threefold peak amplitude

\footnotetext{
*cshua@seu.edu.cn

†fabio.baronio@unibs.it

Published by the American Physical Society under the terms of the Creative Commons Attribution 4.0 International license. Further distribution of this work must maintain attribution to the author(s) and the published article's title, journal citation, and DOI.
}

developing on a finite continuous background, which has served as the basic prototype [24] to explore and confront the fleeting nature of realistic rogue waves [25]. Indeed, the single Peregrine soliton, as a limiting case of the Akhmediev breather or the Kuznetsov-Ma soliton [4,20,26], was observed in many physical settings such as in a water-wave tank [4] or a deep-water flume [5], in optical fibers [20,27], in plasmas [28], and even in irregular oceanic sea states [29]. This Peregrine soliton concept has also been generalized to the coupled nonlinear systems $[30,31]$, exhibiting features that are unavailable to scalar integrable models. As remarkable examples, the allowed Peregrine soliton states can manifest as deep holes for both field components [21], occur in defocusing nonlinear systems [32], coexist with other cousins on the same background [33], or entail ultrastrong peak amplitudes under certain parameter conditions $[34,35]$. In the meantime, the quest of high-order rogue wave dynamics involving interacting Peregrine solitons has also attracted a surge of research activities [36-38], notably on the multiple rogue wave patterns $[39,40]$ and the so-called super rogue wave states [41,42]. All these studies on deterministic rogue waves, either theoretical or experimental, have greatly enriched our understanding on the nature of rogue waves, whereby one can observe, generate, and utilize the otherwise elusive and indocile rogue waves in a practical environment $[43,44]$.

However, some fundamental issues related to rogue wave excitation and generation remain unclear. Among these issues, we here address the question of whether the coexistence of several rogue wave solutions can be considered as a general situation, which implies extended favorable regions of parameters and initial conditions, or whether such coexistence 
would be rather exceptional $[33,45]$. It is interesting to note the analogy between the previous question and that of the existence of bistable solitons [46-48]. Rogue wave coexistence means that at least two different rogue wave states could develop and interact on the same background, which is a dynamical consideration practically untouched upon by now, to say nothing of the underlying physics responsible for such coexisting dynamics. Moreover, most previous studies have been subject to continuous backgrounds [2,7,14], which becomes an important limitation when a vector nonlinear system is adopted to generate rogue waves, as the interference between two continuous-wave (cw) fields can create a periodic background. In history, it was basically the appearance of such periodic fringes caused by interference that established the wave nature of light. Therefore, it is important not only to generalize dynamical systems from scalar to vector cases, but also to generalize the background conditions according to the new possibilities presented in the extended system. Accordingly, can coexisting rogue wave dynamics occur on a periodic background as well as on a continuous one? If so, is the coexistence a universal feature of vector rogue waves that would be present over a wide range of parameters?

In this article, we will address these interesting yet open questions, by investigating the coexisting rogue wave dynamics in a nonlinear two-wave interference system governed by the vector Chen-Lee-Liu (CLL) nonlinear Schrödinger (NLS) equation [49-51], which is an integrable extension of the scalar counterpart [52-55]. We report the exact rogue wave solutions describing the fundamental (first-order) Peregrine solitons and their higher-order complexes on the more general periodic backgrounds. Here, the background periodicity originates from the interference between two $\mathrm{cw}$ fields [56,57], and is therefore of trigonometrical origin, different from the cnoidal-wave periodicity occurring in the scalar nonlinear systems [58-61], which generally involves the doubly periodic Jacobian or Weierstrass elliptic functions [62,63]. It is revealed that coexisting rogue waves can occur in a particularly broad range of parameters, which leads us to qualify this coexistence as omnipresent. Interestingly, such omnipresent coexistence can be anticipated by the appearance of two peaks in the modulation instability (MI) spectrum, whose map is typically composed of one sub-baseband and one passband spectral region. At last, we numerically confirm the robustness of the coexisting Peregrine solitons against initial white noise as well as their occurrence in a turbulent wave field caused by MI.

\section{VECTOR CLL MODEL AND GENERAL ROGUE WAVE SOLUTIONS}

We consider the vector CLL-NLS model that governs the mixing of two fundamental-frequency (FF) pulses in a quadratic nonlinear crystal via a type II highly phasemismatched second-harmonic generation process [49-51]. In dimensionless form, it reads

$$
\begin{aligned}
& i u_{1 z}+\frac{\sigma}{2} u_{1 t t}+\left(\left|u_{1}\right|^{2}+\left|u_{2}\right|^{2}\right)\left(u_{1}+i \gamma u_{1 t}\right)=0, \\
& i u_{2 z}+\frac{\sigma}{2} u_{2 t t}+\left(\left|u_{1}\right|^{2}+\left|u_{2}\right|^{2}\right)\left(u_{2}+i \gamma u_{2 t}\right)=0,
\end{aligned}
$$

where $u_{1,2}(z, t)$ are the normalized complex envelopes of the two interacting FF optical fields, with $z$ and $t$ being the propagation distance and the retarded time, respectively. As usual, the subscripts $z$ and $t$ stand for the partial derivatives. Here, the parameter $\sigma= \pm 1$ associated to the second term represents the type of dispersion, that is, +1 for anomalous dispersion and -1 for normal dispersion. The third term combines the Kerr nonlinearity, whose coefficient has been normalized to unity, and the self-steepening effect, whose strength is defined by the parameter $\gamma$ [64]. In many physical scenarios, the self-steepening term arises from the first-order correction to the slowly varying envelope approximation and often comes along with the effects of third-order dispersion and selffrequency shift [65]. However, in some specific situations, e.g., in quadratic nonlinear media, the controllable property of the self-steepening effect enables it to dominate over these synergetic effects, even over the self-phase modulation effect [49,51]. As one can see, in the absence of the self-steepening effect, the above vector model boils down to the Manakov system [1,30], which is frequently used to model nonlinear vector dynamics such as the propagation of optical pulses in randomly birefringent fibers [65], the formation of spatial solitons in planar waveguides [66], or the crossing sea states occurring in the open ocean [67]. As an important generalization of the Manakov system, the vector CLL-NLS model can offer more accurate description of the wave evolutions in such different nonlinear media.

Because of complete integrability [68], Eqs. (1) and (2) can be cast into a $3 \times 3$ linear eigenvalue problem:

$$
\mathrm{R}_{t}=\mathrm{UR}, \quad \mathrm{R}_{z}=\mathrm{VR},
$$

where $\mathrm{R}=[r, s, w]^{\top}$ is the eigenfunction ( $\mathrm{T}$ represents the transpose and $r, s$, and $w$ are functions of $z$ and $t$ ) and the matrices $\mathrm{U}$ and $\mathrm{V}$ are given by

$$
\begin{aligned}
\mathrm{U}= & -\frac{i(\lambda-\sigma) \sigma_{3}}{2 \sigma \gamma}+\frac{\sqrt{\lambda}}{\sigma} \mathrm{Q}+\frac{i \gamma}{\sigma} \mathrm{KQ}^{2}, \\
\mathrm{~V}= & \left.-\frac{i(\lambda-\sigma)^{2} \sigma_{3}}{4 \sigma \gamma^{2}}+\frac{i \gamma^{2}}{2 \sigma} \mathrm{KQ}^{4}-\frac{\gamma}{2} \mathrm{~K}_{(} \mathrm{QQ}_{t}-\mathrm{Q}_{t} \mathrm{Q}\right) \\
& +\frac{\sqrt{\lambda}}{2 \sigma}\left(\frac{\lambda-\sigma}{\gamma} \mathrm{Q}-i \sqrt{\lambda} \sigma_{3} \mathrm{Q}^{2}+\gamma \mathrm{Q}^{3}+i \sigma \sigma_{3} \mathrm{Q}_{t}\right),
\end{aligned}
$$

with $\mathrm{K}=\operatorname{diag}(0,1,1), \sigma_{3}=\operatorname{diag}(1,-1,-1)$, and

$$
\mathrm{Q}=\left[\begin{array}{ccc}
0 & u_{1} & u_{2} \\
-u_{1}^{*} & 0 & 0 \\
-u_{2}^{*} & 0 & 0
\end{array}\right] .
$$

Here $\lambda$ is the complex spectral parameter and the asterisk denotes the complex conjugation. As one can verify, Eqs. (1) and (2) can be readily reproduced from the compatibility condition $\mathrm{U}_{z}-\mathrm{V}_{t}+\mathrm{UV}-\mathrm{VU}=0$. We should point out that the above Lax pair form (3) for the vector CLL-NLS equation is new and cannot be simply generalized from the $2 \times 2$ Lax pair form provided in Ref. [42] for the scalar CLL-NLS equation.

On an analytical level, there is a straightforward procedure, known as Darboux dressing technique, to derive the new solutions (e.g., solitons, breathers, or rogue waves) from the old ones (e.g., zero or plane waves) [69,70]. In plain terms, suppose first $u_{1,2}$ are the seeding solutions, and then one can 
solve the Lax pair (3) to get the eigenfunction R. In terms of R at given spectral parameter, a dressing operator $\mathrm{D}$ can be constructed, whereby a new eigenfunction $\mathrm{R}^{\prime}=\mathrm{DR}$ is created, which would satisfy the Lax pair (3) of the same form, but with a new pair of potentials $u_{1}^{\prime}$ and $u_{2}^{\prime}$. Lastly, the Darboux transformation formulas that relate the new solutions $u_{1,2}^{\prime}$ to the seeding solutions $u_{1,2}$ can be found from this dressing process. If only the rogue wave states are targeted, one can use a generalized Darboux transformation procedure, which can give the whole hierarchy of solutions without any iteration operation [30,31,42,71].

Following the above procedure, we obtain readily the general $n$ th-order rogue wave solutions of Eqs. (1) and (2), which can be written in a compact matrix form:

$$
\begin{aligned}
& u_{1, \mathrm{cw}}^{[n]}=u_{10}\left(1-\frac{i}{\left|u_{10}\right|} \mathrm{Y}_{1} \mathrm{M}^{-1} \mathrm{Y}_{2}^{\dagger}\right)\left(\frac{\operatorname{det}(\mathrm{M})}{\operatorname{det}\left(\mathrm{M}^{\dagger}\right)}\right), \\
& u_{2, \mathrm{cw}}^{[n]}=u_{20}\left(1-\frac{i}{\left|u_{20}\right|} \mathrm{Y}_{1} \mathrm{M}^{-1} \mathrm{Y}_{3}^{\dagger}\right)\left(\frac{\operatorname{det}(\mathrm{M})}{\operatorname{det}\left(\mathrm{M}^{\dagger}\right)}\right) .
\end{aligned}
$$

Here $u_{j 0}(j=1,2)$ are the seeding plane-wave solutions,

$$
u_{j 0}=a_{j} \exp \left(i \omega_{j} t-i k_{j} z\right)
$$

with their amplitudes $\left(a_{j}\right)$, wave numbers $\left(k_{j}\right)$, and frequencies $\left(\omega_{j}\right)$ obeying the dispersion relations

$$
k_{j}=\left(a_{1}^{2}+a_{2}^{2}\right) v_{j}+\frac{1}{2} \sigma \omega_{j}^{2}, \quad v_{j}=\gamma \omega_{j}-1,
$$

which would serve as the continuous backgrounds to support rogue waves. The $1 \times n$ row vectors $\mathrm{Y}_{j}$ and the $n \times n$ square matrix $\mathrm{M}$ can be exactly determined from the Taylor expansions of $\Phi(\lambda)=G^{-1} R$ and $\Phi^{\dagger} X \Phi /\left(\lambda-\lambda^{*}\right)$ at the given point $\lambda=\lambda_{0}$, respectively, where $\mathrm{G}=\operatorname{diag}\left(1, u_{10}^{*} / a_{1}, u_{20}^{*} / a_{2}\right)$ and $X=\operatorname{diag}\left(\sqrt{\lambda^{*}}, \sqrt{\lambda}, \sqrt{\lambda}\right)$ are diagonal matrices. One can refer to Appendix A for the technical details of derivations of these matrices. As usual, the symbol $\dagger$ denotes the complex conjugate transpose and "det" stands for the determinant of a matrix.

Quite interestingly, after a simple $\mathrm{SO}(2)$ rotational group operation on the above rogue wave solutions, we can get the general rogue wave solutions developed on the periodic backgrounds,

$$
\begin{aligned}
& u_{1}^{[n]}=u_{1, \mathrm{cw}}^{[n]} \cos \phi-u_{2, \mathrm{cw}}^{[n]} \sin \phi, \\
& u_{2}^{[n]}=u_{1, \mathrm{cw}}^{[n]} \sin \phi+u_{2, \mathrm{cw}}^{[n]} \cos \phi,
\end{aligned}
$$

where $\phi$ is a real free parameter denoting the rotation angle. As one can check, the rogue wave solutions (4) and (5) on the continuous backgrounds are the special case of these periodicbackground solutions with $\phi=0$. Actually, if taking a more general SU(2) group operation, one can further generalize the solutions (8) and (9) into

$$
\begin{aligned}
& u_{1}^{[n]}=u_{1, \mathrm{cw}}^{[n]}\left(\cos \phi-i n_{3} \sin \phi\right)-u_{2, \mathrm{cw}}^{[n]}\left(i n_{1}+n_{2}\right) \sin \phi, \\
& u_{2}^{[n]}=u_{1, \mathrm{cw}}^{[n]}\left(-i n_{1}+n_{2}\right) \sin \phi+u_{2, \mathrm{cw}}^{[n]}\left(\cos \phi+i n_{3} \sin \phi\right),
\end{aligned}
$$

where $n_{1}, n_{2}$, and $n_{3}$ are the directional cosines of an arbitrary unit vector $\hat{\mathrm{n}}$ (i.e., $n_{1}^{2}+n_{2}^{2}+n_{3}^{2}=1$ ). It is easy to find that the latter generalized solutions, although involving two more free parameters, show no more significantly different solution properties than the former ones, of course within the framework of our integrable equation model. Hence, in the following, we are only concerned with the solutions (8) and (9) for the sake of brevity, particulary with an emphasis on the fundamental rogue wave solutions as well as their intriguing coexisting dynamics on a periodic background.

\section{FUNDAMENTAL PEREGRINE SOLITONS AND THEIR OMNIPRESENT COEXISTENCE}

In the simplest case of $n=1$, both rogue wave components, defined by Eqs. (8) and (9), manifest themselves in the form of typical fundamental Peregrine solitons, i.e., exhibit a single localized peak accompanied by two side troughs on a finite continuous or periodic background. After some algebra, we can express these fundamental Peregrine soliton solutions in an explicit form

$$
\begin{aligned}
& u_{1}^{[1]}=u_{10}\left(1+\frac{i G_{1}}{N}\right) \cos \phi-u_{20}\left(1+\frac{i G_{2}}{N}\right) \sin \phi, \\
& u_{2}^{[1]}=u_{10}\left(1+\frac{i G_{1}}{N}\right) \sin \phi+u_{20}\left(1+\frac{i G_{2}}{N}\right) \cos \phi,
\end{aligned}
$$

where $G_{1,2}$ and $N$ are polynomials of $z$ and $t$, given by

$$
\begin{aligned}
N= & \theta^{2}+\sigma^{2} \ell^{2} z^{2}+\frac{\eta^{2}}{4 \alpha \ell^{2}}-\frac{i \gamma}{\alpha}\left(\sigma \gamma \ell^{2} z-\eta \theta\right), \\
G_{j}= & \frac{2\left[\left(m+\omega_{j}\right) \theta-\sigma \ell^{2} z\right]}{\left(m+\omega_{j}\right)^{2}+\ell^{2}}+\frac{2 \gamma\left(\sigma \gamma \ell^{2} z-\eta \theta\right)}{\alpha} \\
& -\frac{i \eta v_{j}}{\alpha\left[\left(m+\omega_{j}\right)^{2}+\ell^{2}\right]},
\end{aligned}
$$

with $\theta=t+(\sigma m-\gamma A) z, A=a_{1}^{2}+a_{2}^{2}, \eta=\gamma m+1$, and $\alpha=\gamma^{2} \ell^{2}+\eta^{2}$. The parameters $m$ and $\ell$ in Eqs. (12) and (13) are the real and imaginary parts of the complex root $\Lambda$ (i.e., $\Lambda=m+i \ell$ ) of the following quartic equation:

$$
\sigma-\frac{a_{1}^{2} v_{1}}{\left(\Lambda+\omega_{1}\right)^{2}}-\frac{a_{2}^{2} v_{2}}{\left(\Lambda+\omega_{2}\right)^{2}}=0 .
$$

We should point out that our Peregrine soliton solutions (10) and (11) have been simplified to their most succinct forms, with their peaks being located on the origin. In the far region from the origin, one can find that the background waves would involve the amplitudes

$$
\begin{aligned}
& \left|u_{1}^{\mathrm{bg}}\right|=\sqrt{a_{1}^{2} \cos ^{2} \phi+a_{2}^{2} \sin ^{2} \phi-\sin (2 \phi) a_{1} a_{2} \cos \varphi}, \\
& \left|u_{2}^{\mathrm{bg}}\right|=\sqrt{a_{1}^{2} \sin ^{2} \phi+a_{2}^{2} \cos ^{2} \phi+\sin (2 \phi) a_{1} a_{2} \cos \varphi},
\end{aligned}
$$

where $\varphi=\delta[(A \gamma+\sigma \kappa / 2) z-t], \quad \kappa=\omega_{1}+\omega_{2}, \quad$ and $\delta=$ $\omega_{1}-\omega_{2}$. It is seen that these backgrounds exhibit periodicity, depending not only on the temporal beat frequency that is equal to $\delta$ as well as the transversal velocity that is equal to $v=t / z=A \gamma+\sigma \kappa / 2$, but also on the rotation angle $\phi$, which defines the modulation depth of the background fields. It follows that the average modulation depth in a large enough time interval is equal to $d_{1}=\sqrt{a_{1}^{2} \cos ^{2} \phi+a_{2}^{2} \sin ^{2} \phi}$ for the $u_{1}$ component and $d_{2}=\sqrt{a_{1}^{2} \sin ^{2} \phi+a_{2}^{2} \cos ^{2} \phi}$ for the $u_{2}$ one. 
Hence, on the origin, the ratios of the peaks to the average background height for these periodic Peregrine solitons can be evaluated by

$$
\begin{aligned}
& f_{u_{1}}=\frac{\left|u_{1}(0,0)\right|}{d_{1}}=\frac{\left|a_{1} f_{1} \cos \phi-a_{2} f_{2} \sin \phi\right|}{\sqrt{a_{1}^{2} \cos ^{2} \phi+a_{2}^{2} \sin ^{2} \phi}}, \\
& f_{u_{2}}=\frac{\left|u_{2}(0,0)\right|}{d_{2}}=\frac{\left|a_{1} f_{1} \sin \phi+a_{2} f_{2} \cos \phi\right|}{\sqrt{a_{1}^{2} \sin ^{2} \phi+a_{2}^{2} \cos ^{2} \phi}},
\end{aligned}
$$

where $f_{1,2}$ are the enhancement factors occurring at $\phi=0$ for two field components, given by

$$
f_{j}=1+\frac{4 v_{j} \ell^{2}}{\eta\left[\left(m+\omega_{j}\right)^{2}+\ell^{2}\right]} \quad(j=1,2) .
$$

Before proceeding, let us first inspect the special case of zero frequency difference, namely, $\delta=\omega_{1}-\omega_{2}=0$. In this situation, one can derive from Eq. (14) that $m=-\omega_{1}$ and $\ell= \pm \sqrt{-\sigma v_{1} A} / \sigma$, with $\sigma v_{1}<0$. It turns out that the Peregrine soliton solutions (10) and (11) can then become decoupled completely, exhibiting no periodicity in backgrounds and a fixed threefold peak amplitude for each component, as occurred in the scalar CLL-NLS scenario [42,54]. This suggests that the Peregrine solitons on a periodic background are unable to exist in the vector system with zero frequency difference. Hence, in the following, we assume $\delta \neq 0$ so that the intriguing rogue wave dynamics can manifest on a periodic background.

When the background amplitudes for two wave components take the form

$$
a_{1}=\sqrt{-\frac{\sigma \delta^{2}}{v_{1}}}, \quad a_{2}=\sqrt{-\frac{\sigma \delta^{2}}{v_{2}}}
$$

where $\sigma v_{1,2}<0$, the quartic equation (14) will have one conjugated pair of complex roots, yielding $m=-\kappa / 2$ and $\ell=\sqrt{3} \delta / 2$. Here we have dropped the \pm sign from the $\ell$ formula, as the solutions depend on $\ell^{2}$ only. By inserting these $m$ and $\ell$ values into Eqs. (12) and (13) and then into Eqs. (10) and (11), fundamental Peregrine soliton states on a periodic background can be obtained. Figure 1 displays such Peregrine soliton states in the anomalous dispersion regime, with $\phi$ evolving from 0 to $\pi / 4, \pi / 3$, and finally to $\pi / 2$. Conforming to the amplitude condition (20), we selected here a set of background parameters $\gamma=1, \omega_{1}=-1, \omega_{2}=0, a_{1}=\sqrt{2} / 2$, and $a_{2}=1$. It is shown that, at $\phi=0$, the rogue wave components take the typical Peregrine soliton structures on a continuous background [see Figs. 1(a) and 1(b)], then evolve into the Peregrine solitons with a periodic background at $\phi=\pi / 4$ [see Figs. 1(c) and 1(d)] and at $\phi=\pi / 3$ [see Figs. 1(e) and 1(f)], and finally return to the continuous-background Peregrine soliton states at $\phi=\pi / 2$ [see Figs. 1(g) and 1(h)], which just reverse the positions of those shown in Figs. 1(a) and 1(b). Moreover, as seen in Figs. 1(e) and 1(f), the peak of the Peregrine soliton built on a periodic background can be weakened (enhanced) significantly due to the destructive (constructive) interference of the background fields.

On the other hand, if substituting the $m$ and $\ell$ values into Eqs. (17) and (18), one can find that, due to the presence of the self-steepening effect (i.e., $\gamma \neq 0$ ), the maximum enhancement factor can reach as high as 5 , in either the anomalous
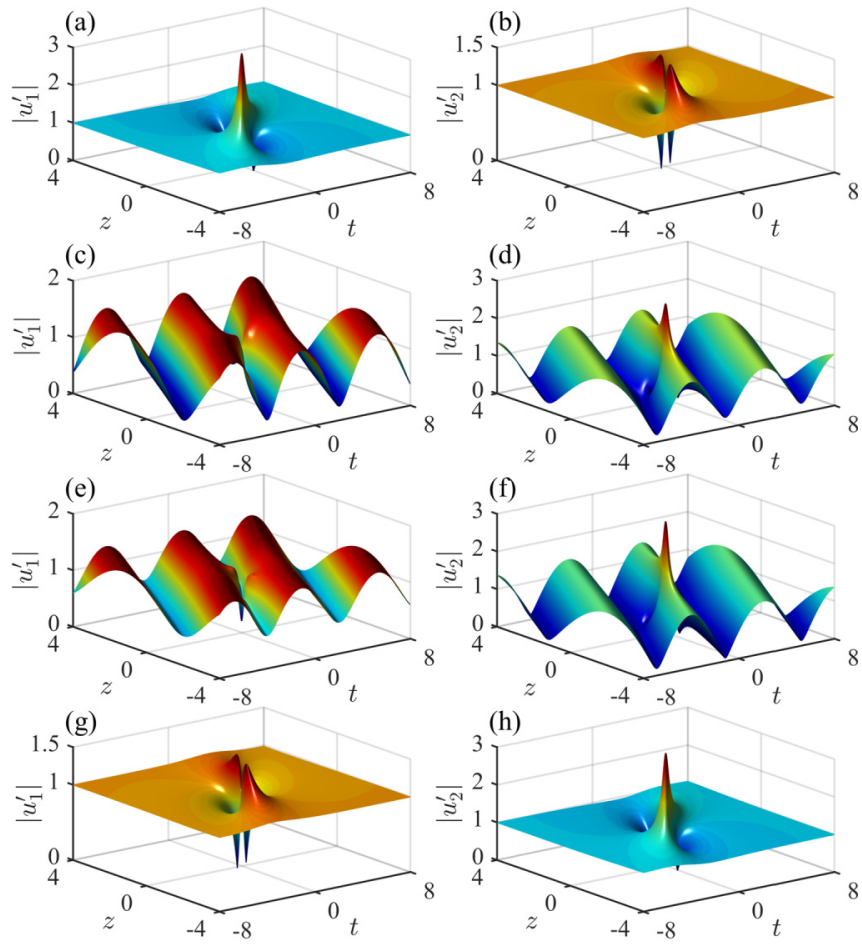

FIG. 1. Evolution of Peregrine soliton states with the rotation angle $\phi$ in the anomalous dispersion regime $(\sigma=1)$, with $\gamma=$ $1, \omega_{1}=-1, \omega_{2}=0, a_{1}=\sqrt{2} / 2$, and $a_{2}=1$ (yielding $m=1 / 2$ and $\ell=-\sqrt{3} / 2$ ). (a),(b) $\phi=0$; (c),(d) $\phi=\pi / 4$; (e),(f) $\phi=\pi / 3$; (g),(h) $\phi=\pi / 2$. Here and in what follows, all the rogue wave components plotted have been normalized to their respective average background heights, i.e., $\left|u_{1}^{\prime}\right|=\left|u_{1}\right| / d_{1}$ and $\left|u_{2}^{\prime}\right|=\left|u_{2}\right| / d_{2}$, unless otherwise stated.

or normal dispersion situation, as revealed in Ref. [34]. To illustrate this, we consider the normal dispersion regime and select a different set of parameters that meets Eq. (20), i.e., $\gamma=1, \omega_{1}=19 / 10, \omega_{2}=13 / 10, a_{1}=\sqrt{10} / 5$, and $a_{2}=$ $\sqrt{30} / 5$. The results are shown in Fig. 2, suggesting that the Peregrine solitons on a periodic background may have a peak amplitude higher than the threefold average background height as well as done on a continuous background [see Figs. 2(a) and 2(c)]. Generally, this enhancement is obtained at the expense of the falling off of the other rogue wave component in the center, as seen in Figs. 2(b) and 2(d). One possible practical significance of this anomaly of the vector Peregrine soliton is that the fundamental-mode rogue waves can be created even from a weak noisy background field, as indicated in Refs. [34,35]. Actually, a rogue wave of a more than 100 intensity factor has been confirmed in particle-in-cell (PIC) simulations of the gyrotron microwave turbulence [72].

Of particular interest is the case when the quartic equation (14) has two different conjugated pairs of complex roots. As such, one can find that each rogue wave component may exhibit two different spatiotemporal structures that coexist on the same periodic background [33]. By inspecting the nature of roots of Eq. (14), the parameter condition for coexisting rogue waves can be expressed as

$$
\sigma v_{1,2}<0, \quad \delta \neq 0, \quad \text { and } \varpi \neq 0 \text { or } \iota \neq-2 \delta^{2} \sigma,
$$



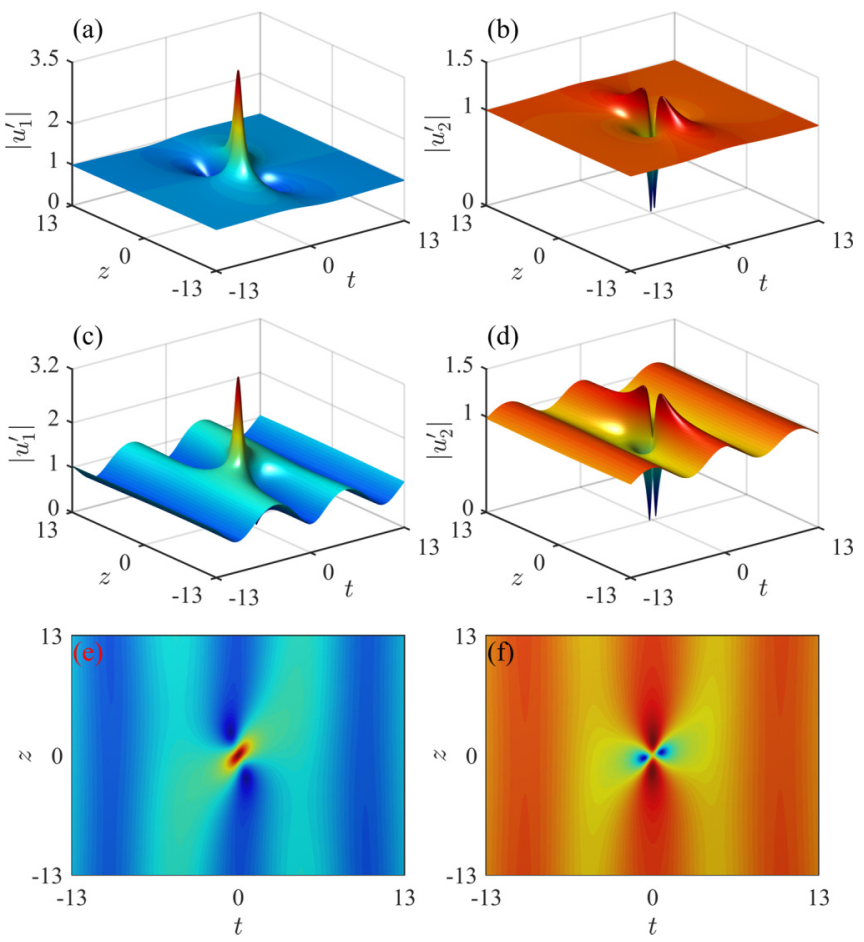

FIG. 2. Typical Peregrine soliton states on (a),(b) the continuous background $(\phi=0)$ and (c),(d) the periodic background $(\phi=\pi / 18)$ in the normal dispersion regime $(\sigma=-1)$, with $\gamma=1, \omega_{1}=19 / 10$, $\omega_{2}=13 / 10, a_{1}=\sqrt{10} / 5$, and $a_{2}=\sqrt{30} / 5$ (yielding $m=-8 / 5$ and $\ell=3 \sqrt{3} / 10$ ). Panels (e) and (f) show the contour plots of (c) and (d), respectively.

where $\iota=a_{1}^{2} v_{1}+a_{2}^{2} v_{2}$ and $\varpi=a_{1}^{2} v_{1}-a_{2}^{2} v_{2}$. This actually represents the great majority of scenarios, as the condition (21) implies that any background amplitudes other than Eq. (20) will be favorable for rogue wave coexistence, provided that $\sigma v_{1,2}<0$ is met. For example, if we simply take half of the background amplitudes (20), viz.,

$$
a_{1}=\frac{1}{2} \sqrt{-\frac{\sigma \delta^{2}}{v_{1}}}, \quad a_{2}=\frac{1}{2} \sqrt{-\frac{\sigma \delta^{2}}{v_{2}}},
$$

Eq. (14) can be easily solved, yielding two different sets of $(m, \ell)$ values, namely, $\left(m=-\kappa / 2+12^{1 / 4} \delta / 4, \ell=\right.$ $\left.12^{1 / 4} \delta / 4\right)$ and $\left(m=-\kappa / 2-12^{1 / 4} \delta / 4, \ell=12^{1 / 4} \delta / 4\right)$. When these $(m, \ell)$ sets are substituted into Eqs. (10) and (11), different rogue wave structures would appear. Figure 3 shows the coexistence of two different Peregrine soliton states on a continuous background $(\phi=0)$ in the normal dispersion regime, where the same set of background parameters $\omega_{1}=19 / 10$, $\omega_{2}=11 / 10, a_{1}=2 \sqrt{10} / 15$, and $a_{2}=2 \sqrt{10} / 5$ was used. It is seen that, on such continuous backgrounds, the two rogue wave components may exhibit the bright-dark structure [see Figs. 3(a) and 3(b)] or the bright-bright one [see Figs. 3(c) and $3(\mathrm{~d})$ ], depending on the values of $(m, \ell)$ used. More interestingly, in the latter bright-bright case, one component may have a peak amplitude much higher than the threefold background height $\left(f_{u_{1}} \approx 4.3\right)$, without leading to an obvious sinkage on the other component $\left(f_{u_{2}} \approx 2.2\right)$, different from those shown in Figs. 3(a) and 3(b) or in Fig. 2.
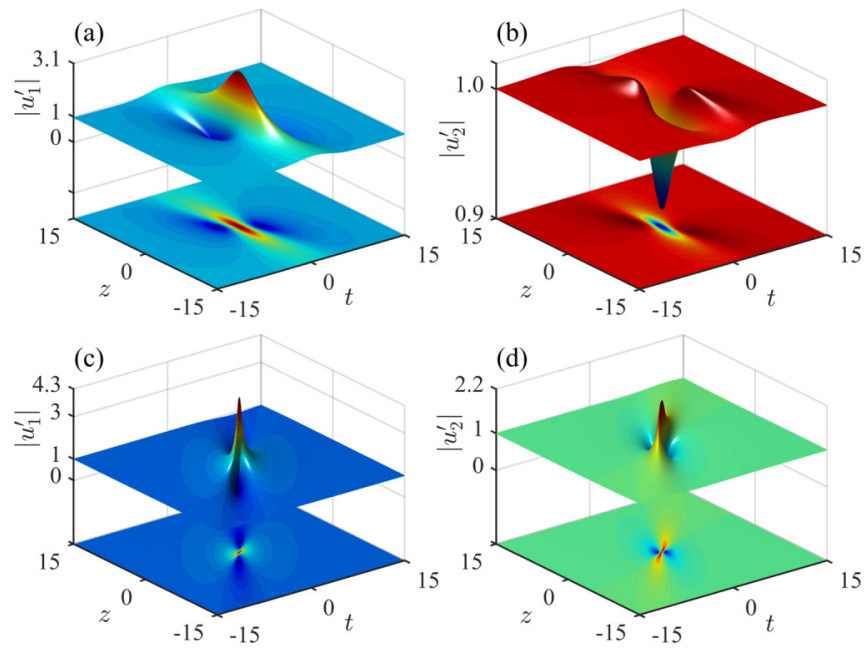

FIG. 3. Surface (top) and contour (bottom) plots of two families of coexisting Peregrine solitons on the same continuous background in the normal dispersion regime $(\sigma=-1)$, with $\gamma=1, \omega_{1}=$ $19 / 10, \omega_{2}=11 / 10, a_{1}=2 \sqrt{10} / 15$, and $a_{2}=2 \sqrt{10} / 5$. (a),(b) $m=$ $-3 / 2-12^{1 / 4} / 5$ and $\ell=12^{1 / 4} / 5$; (c),(d) $m=-3 / 2+12^{1 / 4} / 5$ and $\ell=12^{1 / 4} / 5$.

Such coexisting behaviors can occur as well for the Peregrine solitons on the periodic backgrounds. Figure 4 demonstrates the coexistence of Peregrine solitons at $\phi=$ $\pi / 18$, under otherwise identical parameters as in Fig. 3. It is
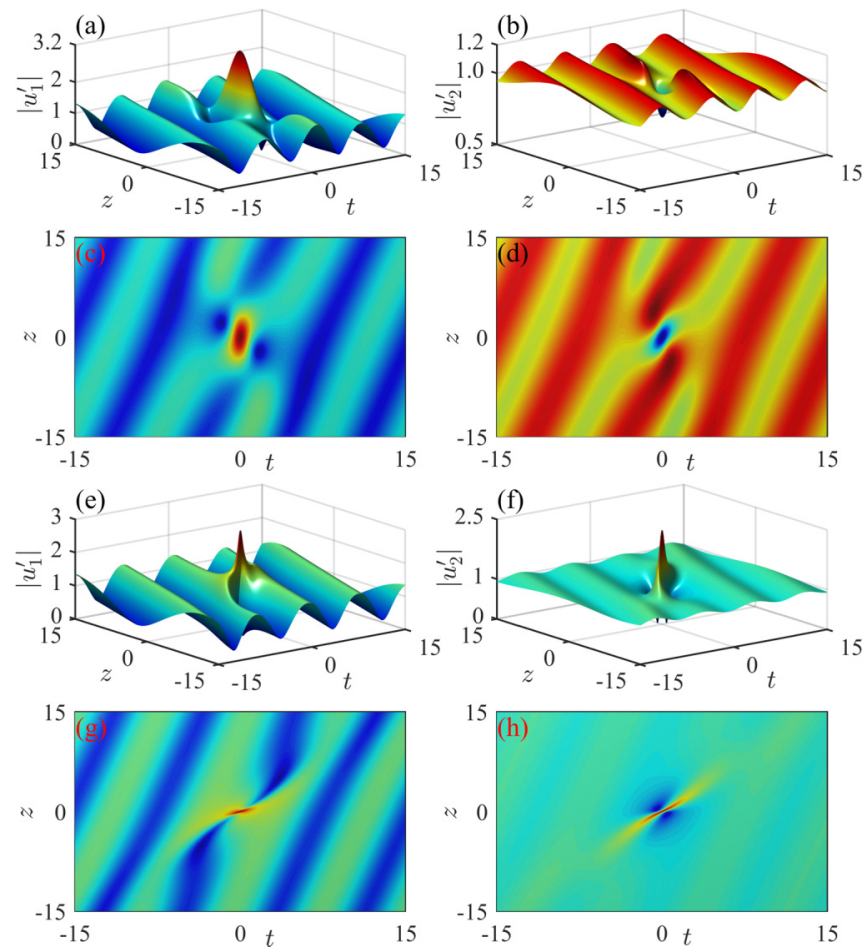

FIG. 4. Coexistence of two different types of Peregrine solitons on the same periodic background $(\phi=\pi / 18)$, using the same parameters as in Fig. 3, with (a),(b),(e),(f) showing the surface plots of the normalized amplitudes, while with (c),(d), (g),(h) showing the corresponding contour plots. 

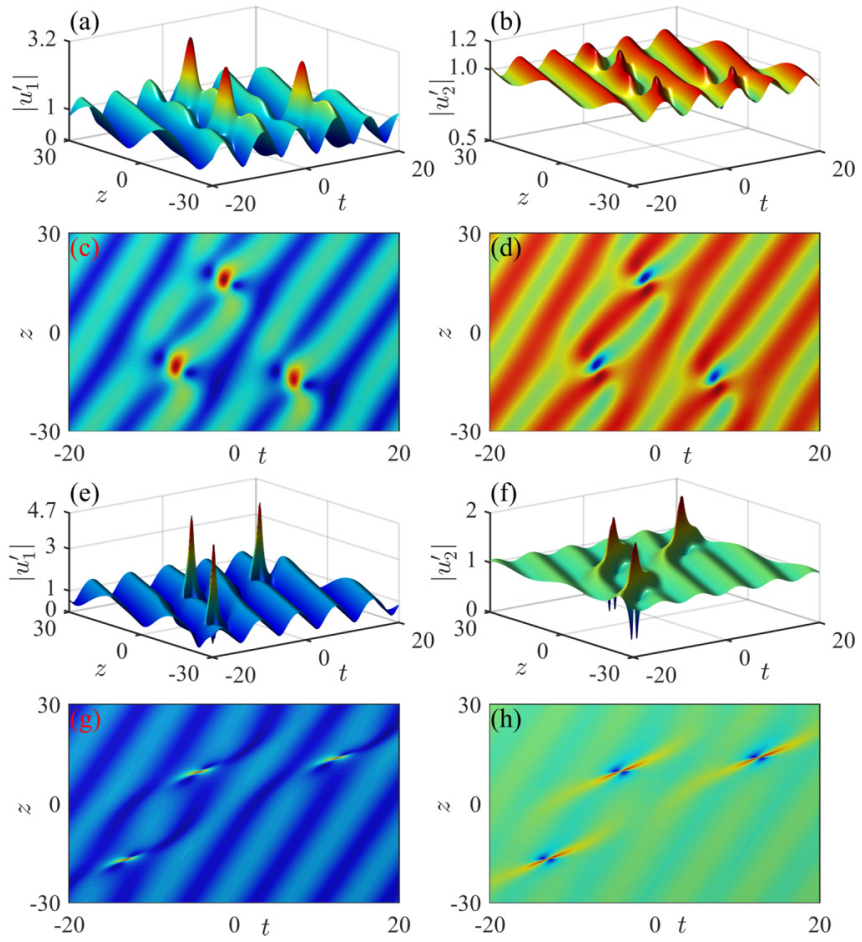

FIG. 5. Coexistence of two families of triplet rogue waves on the same periodic background $(\phi=\pi / 18)$, under the same parameter condition as in Fig. 4, with (a),(b),(e),(f) showing the surface plots of the normalized amplitudes, while with (c),(d),(g),(h) showing the corresponding contour plots.

clear that, in this case, both the bright-dark [see Figs. 4(a)4(d)] and bright-bright [see Figs. 4(e)-4(h)] patterns remain visible on the periodic backgrounds. Moreover, the peak amplitudes of these coexisting Peregrine solitons still can grow beyond the threefold average background height, as implied in Figs. 4(a) and 4(e). This suggests the observability of such coexistence in the laboratory setting, for given appropriate parameter conditions. In addition, the importance of the role played by the self-steepening term can also be seen from Eq. (21). It turns out that the self-steepening effect can allow rogue wave coexistence over a broad range of parameters in both the anomalous and normal dispersion regimes, significantly extending the favorable regions of Manakov systems where the coexisting behaviors occur solely in the anomalous dispersion regime $[14,30]$.

The omnipresent coexistence of different rogue wave solutions comes through not only in the fundamental (first-order) Peregrine solitons, but also in higher-order multiple rogue wave patterns. Let us take the triple rogue wave as an example, which, as its name implies, is the one whose pattern is composed of three well-separated Peregrine solitons on a finite background $[22,39,40]$. Their exact dynamics depend on the parameters used in the solutions (8) and (9) with $n=2$. Figure 5 demonstrates such coexisting triple rogue waves on the periodic background in the normal dispersion regime, using the same parameter condition as in Fig. 4, of course with an additional set of structure parameters that is nonessential for showing coexisting dynamics. It is clear that, on the same periodic background, each field component could take two different spatiotemporal patterns, namely, the $u_{1}$ component can take the bright structure shown in Fig. 5(a) or the bright one shown in Fig. 5(e), while the $u_{2}$ component may take a dark structure in Fig. 5(b) or a bright one in Fig. 5(f). Here, for a better view of the complete picture, all figures have been translated along the plane $(z, t)$, with different magnitudes, and therefore the fringes of periodic background shown in Fig. 5(c) have been slightly offset as compared to those shown in Fig. 5(g); so is the case concerning Figs. 5(d) and 5(h). As one can see, the constituents in each triple rogue wave state could take the same Peregrine soliton structure as the corresponding one shown in Fig. 4, implying that the coexisting dynamics is inherent to the vector nonlinear system, no matter whether fundamental Peregrine soliton structures or multiple rogue wave ones are manifested. We will address this issue further in Sec. IV from the perspective of MI.

\section{PERSPECTIVE OF MODULATION INSTABILITY AND NUMERICAL SIMULATIONS}

The coexistence of rogue waves can be seen in a broad range of parameters that fulfills Eq. (21). Now further questions arise as to whether this coexistence can be explained from the perspective of MI [16-18,59,67] and whether coexisting rogue wave dynamics can be generated from the turbulent fields induced by MI. Below we proceed to answer these questions, using the linear stability analysis and extensive numerical simulations.

To begin with, let us add the small amplitude Fourier modes $r_{j}$ and $w_{j}$ to the plane wave solutions (6) and express them as $u_{j}=u_{j 0}\left[1+r_{j} e^{-i \Omega(\varkappa z-t)}+w_{j}^{*} e^{i \Omega\left(\varkappa^{*} z-t\right)}\right](j=1,2)$, where $\Omega$ denotes the frequency offset from the central frequencies caused by small perturbations and $\varkappa$ is a complex wave number induced by perturbations. Similar to the light scattering process [73], we will term $\Omega<0$ the Stokes frequency shift and $\Omega>0$ the anti-Stokes frequency shift. When these perturbed plane-wave solutions are then substituted into Eqs. (1) and (2), followed by the linearization, a system of four coupled linear equations about $r_{1,2}$ and $w_{1,2}$ can be obtained, which will have a nontrivial solution only when $\varkappa$ satisfies the dispersion relation:

$$
\begin{aligned}
\Pi^{4}= & \left(\frac{\Omega^{2}+\delta^{2}}{2}+\sigma \iota\right) \Pi^{2}+\varpi \delta \Pi \\
& -\frac{\left(\Omega^{2}-\delta^{2}\right)\left(\Omega^{2}-\delta^{2}+4 \sigma \iota\right)}{16},
\end{aligned}
$$

where $\Pi=\varkappa-\gamma A-\kappa \sigma / 2$ and we let $\sigma^{2}=1$ by assumption. Obviously, only when $\varkappa$ or $\Pi$ is a complex number with nonzero imaginary part does the MI occur. Therefore, we define the gain factor $\gamma_{h}=|\Omega \operatorname{Im}(\Pi)|$ so as to quantize the strength of MI caused by external perturbations or by spontaneous noises [14].

As can be seen, a full understanding of the MI gain requires plotting $\gamma_{h}$ in five-dimensional phase space, i.e., with respect to five variables $\delta, \kappa, a_{1}, a_{2}$, and $\Omega$. Here, for clarity, we only present the gain maps versus $\Omega$ and $\delta$, solely intended for the dynamics of coexisting rogue waves shown in Figs. 3, 4, and 5. To gain an insight into the relation between MI and the coexisting rogue waves, we adopt two strategies to plot the 

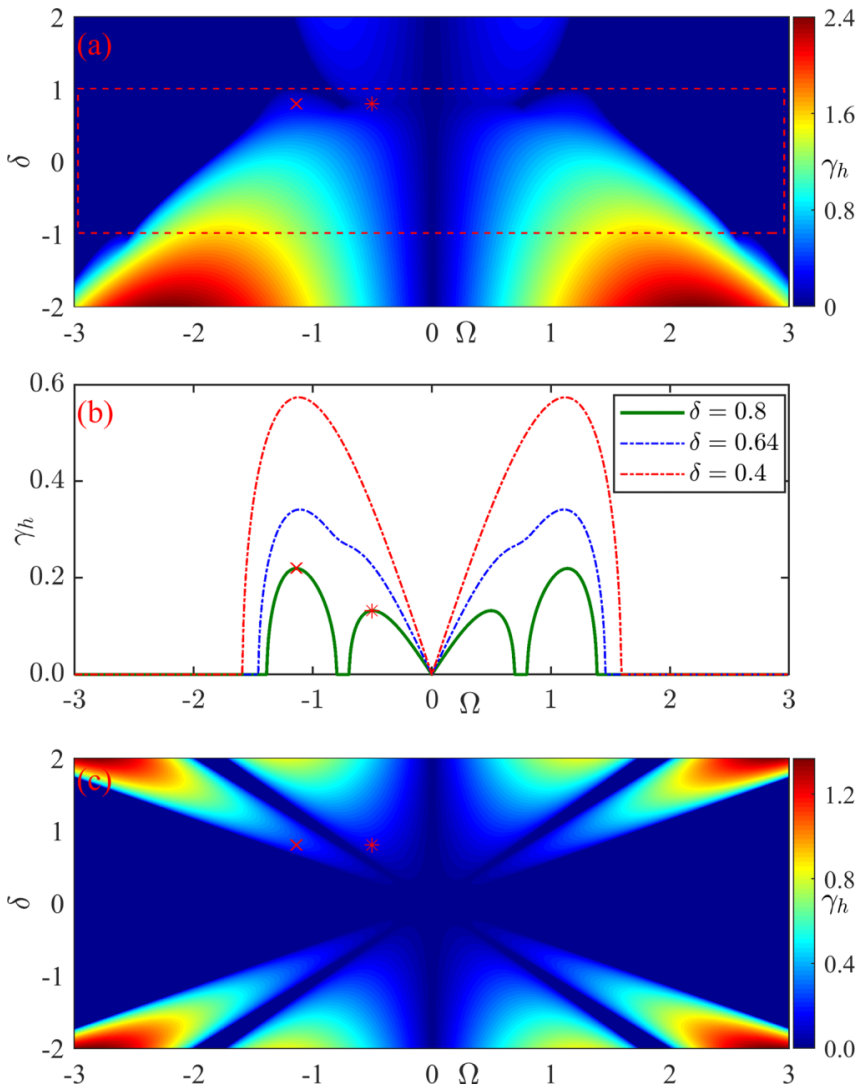

FIG. 6. Maps of the MI gain $\gamma_{h}$ versus $\Omega$ and $\delta$ in the normal dispersion regime $(\sigma=-1)$ for $\kappa=3$ and $\gamma=1$ : (a) letting $a_{1}=$ $2 \sqrt{10} / 15$ and $a_{2}=2 \sqrt{10} / 5$ or (c) letting $a_{1,2}$ vary but follow the condition (22). Panel (b) shows different gain profiles versus $\Omega$ by traversing the MI map (a) at $\delta=0.8,0.64$, and 0.4 , respectively. The red crosses denote the maximum gain $\gamma_{h}^{\max }=0.22$ occurring at $\Omega=$ -1.14 , while the stars denote the second gain peak at $\Omega=-0.5$, both with $\delta=0.8$.

MI gain map. One strategy is to keep the background amplitudes unchanged, i.e., $a_{1}=2 \sqrt{10} / 15$ and $a_{2}=2 \sqrt{10} / 5$, which yields the MI map in Fig. 6(a). In this map, we find that the gain distribution is asymmetrical with respect to $\delta$ and the coexisting rogue waves would be admissible within the range $-1<\delta<1$ (see the red dashed box region), according to the parameter condition (21). We then select several different $\delta$ values and plot the related gain profiles by traversing Fig. 6(a) at these values. It is seen that, at the specific value $\delta=0.8$ that brings us with Figs. 3, 4, and 5, the gain profile, indicated by the green solid curve, consists of two gain bands in the Stokes frequency shift region $(\Omega<0)$ or in the anti-Stokes frequency shift region $(\Omega>0)$; the stronger one is located on around $|\Omega|=1.14$, while the weaker one stands on $|\Omega|=0.5$. As $\delta$ decreases, the two gain bands tend to merge, as indicated by the blue and red lines in Fig. 6(b). When $\delta<0.4$, which corresponds to $|\ell / m|<10 \%$, the two spectral peaks completely merge into one. In this case, it is usually hard to see two coexisting Peregrine solitons of comparable sizes on the same background, as one of them will be greatly extended in dimension, and behave more like an ordinary soliton in shape.
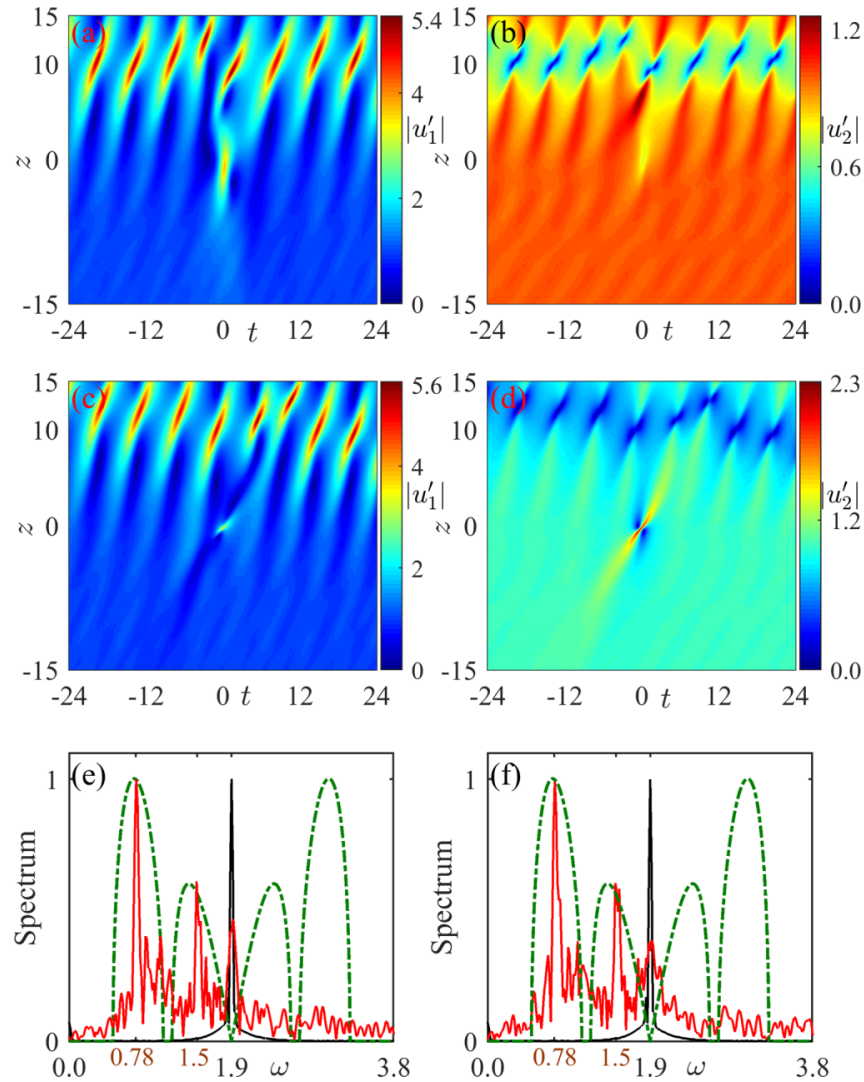

FIG. 7. Panels (a)-(d) show the numerical occurrence of the coexisting Peregrine soliton structures that are demonstrated in Fig. 3 correspondingly, starting from the initial profiles at $z=-15$ perturbed by the white noise. The normalized spectra of the Peregrine solitons versus the angular frequency $\omega$ intended for the $u_{1}$ field are plotted in (e) and (f), which are calculated from (a) and (c), respectively. Black curves: input spectra; red curves: output spectra; green dashed lines: normalized gain profiles obtained analytically from MI map.

The other strategy is to let the amplitudes vary but follow the amplitude condition (22), which would produce the MI map in Fig. 6(c). As one can see, this MI map is now symmetric with respect to $\delta$, and now the MI baseband comprises one sub-baseband and one passband spectral region, resulting in two spectral peaks, for a specific $\delta$ value, in either the Stokes or the anti-Stokes frequency shift region. Typically, at $\delta=0.8$, one can get the gain profile exactly the same as the one shown in Fig. 6(b) (see green curve). Coincidentally, under the amplitude condition (22), coexisting rogue wave dynamics are always allowed. Considering both cases shown in Figs. 6(a) and 6(c), we therefore argue that it is the appearance of this peculiar baseband characteristic in MI maps that may anticipate the occurrence of coexisting rogue waves of comparable size on the same finite background. This is a universal characteristic of coexisting rogue waves and can be found in the MI maps of other vector nonlinear systems that allow the coexisting rogue wave dynamics; see, for example, Fig. 12 in Ref. [14] for the multicomponent long-wave-shortwave system [33].

Actually, our prediction of two spectral peaks in a gain profile associated to the coexisting rogue waves can be confirmed 

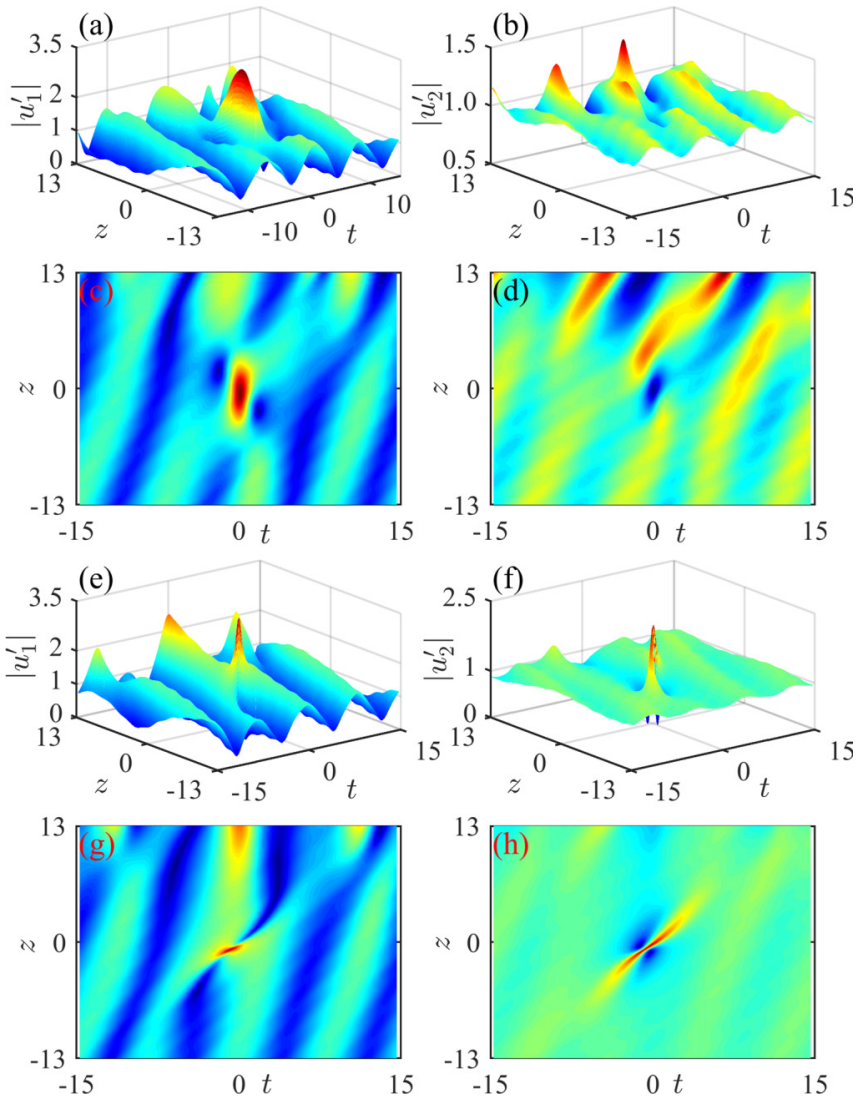

FIG. 8. Numerical simulations of the coexisting Peregrine solitons shown in Fig. 4 perturbed by the white noise, under the otherwise identical parameter conditions as in Fig. 4.

by numerical simulations. For this purpose, the dynamics of coexisting Peregrine solitons shown in Fig. 3 are numerically calculated by means of the split-step Fourier method [34,35], starting from the initial profiles at $z=-15$ perturbed by the white noise. The results are presented in Figs. 7(a)-7(d). It is seen that both rogue wave families on the continuous background can be reproduced clearly, showing an excellent agreement with their analytical solutions. However, shortly after the revival of Peregrine solitons, the MI manifest itself significantly at around $z=10$, whereby a train of periodic waves is induced. We then calculate the input and output spectra of the propagating Peregrine solitons in the presence of MI, by performing Fourier transform of the temporal profile at $z=$ -15 and 15, respectively. Here, we only present in Figs. 7(e) and 7(f) the two spectral results of the $u_{1}$ field, which are calculated from Figs. 7(a) and 7(c), respectively. It is obvious that, at the outset, the spectral peak of the $u_{1}$ field locates on $\omega=1.9=\omega_{1}$ as expected (see black curves), and, however, at the output, due to the MI effect, two additional spectral peaks appear, standing fixedly on $\omega=0.78$ and 1.50 , respectively (see red curves). Strikingly, these numerical results at the output are well consistent with the ones (green curves) derived from our MI analysis, not only on the peak positions but also on the spectrum amplitudes. Note here that the spectral profiles denoted by green dashed curves in Figs. 7(e) and 7 (f) are given in terms of the angular frequency $\omega$, which are exactly the same as the green solid curve in Fig. 6(b), which is

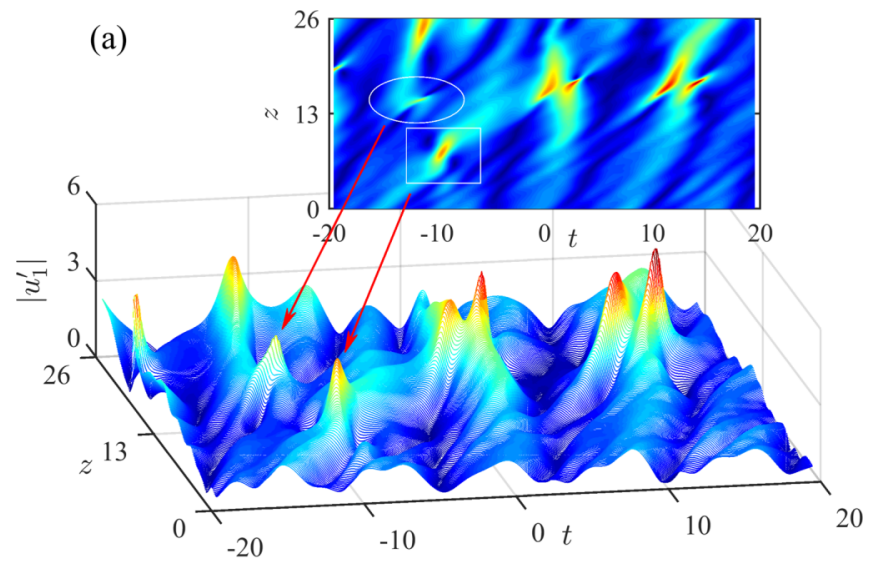

(b)

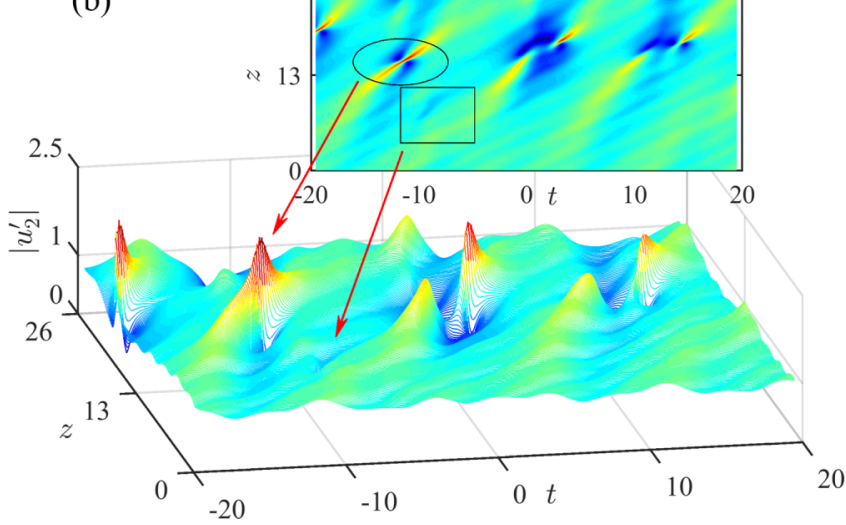

FIG. 9. Numerical excitation of coexisting Peregrine solitons from a turbulent periodic-wave field under otherwise identical parameter conditions as in Fig. 4. The ellipses and boxes in the insets of (a) and (b) have singled out such coexisting Peregrine soliton states in a sea of waves.

given with respect to the frequency detuning $\Omega=\omega-\omega_{1}$. We observe further that, in the MI gain spectrum, only the Stokes frequencies are actually generated. This happens because the anti-Stokes components will experience a strong attenuation in the MI process, as occurred in the stimulated Brillouin scattering process [73].

Given a good comprehension on MI, let us now inspect whether the coexisting rogue waves are stable on a periodic background against spontaneous noises as well and whether they can be generated from the turbulent field caused by the MI. For this end, two numerical tests are performed, solely intended for the analytical results shown in Fig. 4. We first demonstrate in Fig. 8 the numerical results of these coexisting Peregrine solitons, starting from initial profiles at $z=-13$ perturbed by the white noise of moderate strength, under otherwise identical parameter conditions as in Fig. 4. A direct comparison between Figs. 4 and 8 reveals that both families of coexisting Peregrine solitons can be still reproduced numerically, even though now on a periodic background. This suggests that these coexisting rogue waves are robust on a periodic background as well as on a continuous one, despite the onset of MI.

Then, we corroborate by numerical simulations the possibility of excitation of these coexisting Peregrine soliton structures from a turbulent wave field induced by MI, with 
results shown in Fig. 9. In this numerical test, we start from periodic wave solutions [which are a rotation of the planewave solutions (6) by an angle $\phi=\pi / 18$ ] at $z=0$ as initial conditions, perturbed by harmonic waves of random strength. Such noisy background is highly unstable, and soon develops into a turbulent sea of different waves. Strikingly, in this turbulent wave field, the two waves at around $z=6$ and $z=13$ on the left zone can be singled out, which are exactly the Peregrine soliton states shown in Fig. 4 (see the ellipses and boxes in the two insets of Fig. 9). As these coexisting rogue waves can be generated numerically from a noisy background, it is therefore anticipated that such coexisting dynamics can be observed in a laboratorial environment. On the other hand, in such a turbulent field, some higher-amplitude Peregrine rogue waves could manifest as well; see, for example, the rightmost one at around $z=17$ in Fig. 9(a), which can reach a nearly fivefold peak amplitude. This is not surprising because the amplitude condition (22) exploited here could be violated locally, when a strong initial harmonic-wave perturbation is imposed, hence resulting in the appearance of different pairs of coexisting rogue waves that may have an even higher enhancement factor, as seen from Eqs. (17) and (18).

\section{CONCLUSION}

In conclusion, the rogue wave dynamics on a periodic background as well as their omnipresent coexistence were investigated, within the framework of the vector CLL-NLS equation, which can govern the interaction of two FF optical waves in a quadratic nonlinear medium. We presented the exact rogue wave solutions at first and higher orders on the more general periodic backgrounds, by use of a generalized Darboux transformation method. In a strict sense, we identified those solutions of same order that have different spatiotemporal structures, but are built on the same continuous or periodic background as the coexisting rogue waves. Next, we provided the analytical parameter conditions for the coexisting rogue wave dynamics, clearly showing that the coexistence of rogue waves is a universal feature inherent to most vector (or coupled) nonlinear systems [30,31,33]. Such a feature is present at the first rational order (see Fig. 4) and also manifests at higher orders (see Fig. 5).

We further revealed that the self-steepening effect would play an essential role in allowing coexisting rogue wave dynamics over a broad range of parameters in both the normal and anomalous dispersion regimes, in addition to allowing, for specific parameters, the development of peaks with an ultrastrong enhancement of their amplitudes. We related such an omnipresent coexistence of rogue wave solutions to the appearance of two peaks in the Stokes frequency shift region of the MI spectrum, whose map is typically composed of one sub-baseband and one passband spectral region. Lastly, we numerically confirmed the robustness of the coexisting Peregrine solitons against initial noise as well as their excitation from a turbulent wave field caused by MI.

It is anticipated that these findings may shed light on the generation of extreme wave events that results from the interference of multiple continuous wave fields. Moreover, one may apply the overall concepts well to the quasiperiodic background scenario that is caused by the cnoidal-wave periodicity
[58-63] followed by an $\mathrm{SO}(2)$ group operation. Considering that the bistable soliton concept has been used for many practical purposes (e.g., frequency combs [48], optical switching [74], optical communications [75], all-optical processing [76], etc.), we also expect our results to offer an incentive to study the coexisting rogue wave dynamics in similar fields.

\section{ACKNOWLEDGMENTS}

This work was supported by the National Natural Science Foundation of China (Grants No. 11474051 and No. 11974075). Ph.G. acknowledges support from the Engineering and Innovation through Physical Sciences, Hightechnologies, and Cross-disciplinary research (EUR EIPHI) Graduate School (Contract No. ANR-17-EURE-0004). We also appreciate partial support from the Scientific Research Foundation of the Graduate School of Southeast University.

\section{APPENDIX: DERIVATIONS OF SPECIFIC ROW VECTORS $Y_{j}$ AND THE SQUARE MATRIX $M$}

In this Appendix, let us present the technical details for derivations of the row vectors $\mathrm{Y}_{j}$ and the square matrix $\mathrm{M}$ in Eqs. (4) and (5). First, inserting the plane-wave solutions (6) into the Lax pair (3), and solving this system of linear equations, one can get the eigenfunction $\mathrm{R}(\lambda)$,

$$
\begin{gathered}
\mathrm{R}(\lambda)=\mathrm{G} \Phi(\lambda), \\
\Phi(\lambda)=\Gamma_{1}\left[\begin{array}{c}
1 \\
c_{11} \\
c_{21}
\end{array}\right] e^{i \varphi_{1}}+\Gamma_{2}\left[\begin{array}{c}
1 \\
c_{12} \\
c_{22}
\end{array}\right] e^{i \varphi_{2}}+\Gamma_{3}\left[\begin{array}{c}
1 \\
c_{13} \\
c_{23}
\end{array}\right] e^{i \varphi_{3}},
\end{gathered}
$$

where $\Gamma_{j}(j=1,2,3$, the same below $)$ are arbitrary complex constants, $\varphi_{j}=\mu_{j} t+v_{j} z$, and

$$
\begin{gathered}
c_{l j}=-\frac{i a_{l} \gamma \psi_{j}}{\sqrt{\lambda}\left(\psi_{j}-2 \sigma v_{l}\right)} \quad(l=1,2), \\
\psi_{j}=2 \sigma \gamma \mu_{j}-\lambda-\sigma, \\
v_{j}=\frac{2 A \gamma^{2} \lambda-(\lambda-\sigma)^{2}}{4 \sigma \gamma^{2}}+\frac{i \sqrt{\lambda}}{2}\left(\omega_{1} a_{1} c_{1 j}+\omega_{2} a_{2} c_{2 j}\right) \\
+\frac{i \sqrt{\lambda}}{2 \gamma \sigma}\left(A \gamma^{2}-\lambda+\sigma\right)\left(a_{1} c_{1 j}+a_{2} c_{2 j}\right) .
\end{gathered}
$$

The parameters $\mu_{j}$ in Eqs. (A3)-(A5) are the three roots of the cubic equation of $\mu$,

$$
\begin{aligned}
& \left(\mu-\frac{\beta}{2}+\frac{A \gamma}{2 \sigma}-\frac{\kappa}{4}\right)^{3}-3 \varsigma\left(\mu-\frac{\beta}{2}+\frac{A \gamma}{2 \sigma}-\frac{\kappa}{4}\right)+2 \varrho \\
& =0,
\end{aligned}
$$

whose parameters $\beta, \varsigma$, and $\varrho$ are defined by

$$
\begin{aligned}
& \beta=\frac{A \gamma^{2}+\lambda-\sigma}{3 \sigma \gamma}+\frac{\kappa}{6}, \\
& \varsigma=\beta^{2}+\frac{\delta^{2}}{12}-\frac{\iota}{3 \sigma}, \\
& \varrho=\beta^{3}-\frac{\beta\left(2 \iota+\sigma \delta^{2}\right)}{4 \sigma}+\frac{\delta \varpi}{4 \sigma} .
\end{aligned}
$$


Basically, only when the spectral parameter $\lambda$ satisfies

$$
\Delta=\varsigma^{3}-\varrho^{2}=0,
$$

which is the discriminant condition of Eq. (A6), does a hierarchy of rational solutions exist. It follows that, under the condition (A7), Eq. (A6) will admit a double-root solution $\mu_{1}=\mu_{2}=\mu_{0}$, or admit a triple-root solution $\mu_{1}=$ $\mu_{2}=\mu_{3}=\mu_{0}$ when $\varsigma=\varrho=0$ is further met. Solving the complex-coefficient quartic Eq. (A7), one can generally obtain one or two conjugated pairs of complex values of $\lambda$, which we will denote by $\lambda_{0}$ below.

It is easy to check that the factored eigenfunction $\Phi(\lambda)$, given by Eq. (A2), will vanish at the point $\lambda=\lambda_{0}$, under an appropriate choice of $\Gamma_{j}$. Therefore, one can perform the Taylor-series expansion of $\Phi(\lambda)$ at this specific point $\lambda=\lambda_{0}$, from which the row vectors $\mathrm{Y}_{j}$ and the square matrix $\mathrm{M}$ can be obtained. To be specific, there are two ways in doing so. On one side, in the double-root case, letting $\lambda=\lambda_{0}+\chi \epsilon^{2}$ and

$$
\begin{aligned}
& \Gamma_{1}=\frac{1}{2} \sum_{j=1}^{n}\left(\gamma_{2 j-1}+\frac{\gamma_{2 j}}{\epsilon}\right) \epsilon^{2(j-1)}, \\
& \Gamma_{2}=\frac{1}{2} \sum_{j=1}^{n}\left(\gamma_{2 j-1}-\frac{\gamma_{2 j}}{\epsilon}\right) \epsilon^{2(j-1)}, \\
& \Gamma_{3}=0,
\end{aligned}
$$

where $\chi=\lambda_{0}-\lambda_{0}^{*}$ is purely imaginary, $\epsilon$ is a complex perturbation parameter, and $\gamma_{s}$ are arbitrary complex constants termed structure parameters, one can expand $\Phi(\lambda)$ in powers of $\epsilon^{2}$,

$$
\Phi(\lambda)=\Phi^{(0)}+\Phi^{(1)} \epsilon^{2}+\Phi^{(2)} \epsilon^{4}+\cdots+\mathcal{O}\left(\epsilon^{2 n}\right),
$$

where $\mathcal{O}$ means the infinitesimal of the same order.

On the other side, in the triple-root case, which is exactly the case defined by the parameter condition (20), one can set

$$
\begin{aligned}
& \lambda=\lambda_{0}+\chi \epsilon^{3} \text { and } \\
& \Gamma_{1}=\frac{1}{3} \sum_{j=1}^{n}\left(\gamma_{3 j-2}-\frac{\gamma_{3 j-1} \phi_{2}}{\epsilon}+\frac{\gamma_{3 j} \phi_{1}}{\epsilon^{2}}\right) \epsilon^{3(j-1)}, \\
& \Gamma_{2}=\frac{1}{3} \sum_{j=1}^{n}\left(\gamma_{3 j-2}-\frac{\gamma_{3 j-1} \phi_{1}}{\epsilon}+\frac{\gamma_{3 j} \phi_{2}}{\epsilon^{2}}\right) \epsilon^{3(j-1)}, \\
& \Gamma_{3}=\frac{1}{3} \sum_{j=1}^{n}\left(\gamma_{3 j-2}+\frac{\gamma_{3 j-1}}{\epsilon}-\frac{\gamma_{3 j}}{\epsilon^{2}}\right) \epsilon^{3(j-1)},
\end{aligned}
$$

where $\phi_{1}=1 / 2+i \sqrt{3} / 2$ and $\phi_{2}=1 / 2-i \sqrt{3} / 2$, then $\Phi$ can be accurately expanded into

$$
\Phi(\lambda)=\Phi^{(0)}+\Phi^{(1)} \epsilon^{3}+\Phi^{(2)} \epsilon^{6}+\cdots+\mathcal{O}\left(\epsilon^{3 n}\right),
$$

which is a series in powers of $\epsilon^{3}$.

In either case, the row vectors $\mathrm{Y}_{j}$ can then be determined by means of the following matrix relation:

$$
\left[\begin{array}{l}
\mathrm{Y}_{1} \\
\mathrm{Y}_{2} \\
\mathrm{Y}_{3}
\end{array}\right]=\left[\Phi^{(0)}, \Phi^{(1)}, \Phi^{(2)}, \ldots, \Phi^{(n-1)}\right],
$$

while the entries $M_{l j}$ of the square matrix $\mathrm{M}$ can be found through the Taylor expansion

$$
\frac{\Phi^{\dagger} \mathrm{X} \Phi}{\lambda-\lambda^{*}}=\sum_{l j}^{n} M_{l j} \epsilon^{* 2(l-1)} \epsilon^{2(j-1)}+\mathcal{O}\left(|\epsilon|^{4 n}\right),
$$

in the double-root case, or through

$$
\frac{\Phi^{\dagger} \mathrm{X} \Phi}{\lambda-\lambda^{*}}=\sum_{\imath j}^{n} M_{l j} \epsilon^{* 3(l-1)} \epsilon^{3(j-1)}+\mathcal{O}\left(|\epsilon|^{6 n}\right),
$$

in the triple-root case, without any ambiguity.
[1] F. Baronio, A. Degasperis, M. Conforti, and S. Wabnitz, Solutions of the Vector Nonlinear Schrödinger Equations: Evidence for Deterministic Rogue Waves, Phys. Rev. Lett. 109, 044102 (2012).

[2] M. Onorato, S. Residori, U. Bortolozzo, A. Montina, and F. T. Arecchi, Rogue waves and their generating mechanisms in different physical contexts, Phys. Rep. 528, 47 (2013).

[3] J. M. Dudley, F. Dias, M. Erkintalo, and G. Genty, Instabilities, breathers and rogue waves in optics, Nat. Photon. 8, 755 (2014).

[4] A. Chabchoub, N. P. Hoffmann, and N. Akhmediev, Rogue Wave Observation in a Water Wave Tank, Phys. Rev. Lett. 106, 204502 (2011).

[5] G. Dematteis, T. Grafke, M. Onorato, and E. Vanden-Eijnden, Experimental Evidence of Hydrodynamic Instantons: The Universal Route to Rogue Waves, Phys. Rev. X 9, 041057 (2019).

[6] D. R. Solli, C. Ropers, P. Koonath, and B. Jalali, Optical rogue waves, Nature (London) 450, 1054 (2007).

[7] Nonlinear Guided Wave Optics: A testbed for extreme waves, edited by S. Wabnitz (IOP Publishing, Bristol, 2017).
[8] Z. Wang, K. Nithyanandan, A. Coillet, P. Tchofo-Dinda, and P. Grelu, Buildup of incoherent dissipative solitons in ultrafast fiber lasers, Phys. Rev. Research 2, 013101 (2020).

[9] P.-C. Lin and L. I, Synchronization of multiscale waveform focusing for rogue wave generation in dust acoustic wave turbulence, Phys. Rev. Research 2, 023090 (2020).

[10] M. G. Copus and R. E. Camley, Creation of magnetic rogue waves, Phys. Rev. B 102, 220410(R) (2020).

[11] Y. V. Bludov, V. V. Konotop, and N. Akhmediev, Matter rogue waves, Phys. Rev. A 80, 033610 (2009).

[12] D. Mihalache, Localized structures in optical and matter-wave media: A selection of recent studies, Rom. Rep. Phys. 73, 403 (2021).

[13] G. Marcucci, D. Pierangeli, and C. Conti, Theory of Neuromorphic Computing by Waves: Machine Learning by Rogue Waves, Dispersive Shocks, and Solitons, Phys. Rev. Lett. 125, 093901 (2020).

[14] S. Chen, F. Baronio, J. M. Soto-Crespo, P. Grelu, and D. Mihalache, Versatile rogue waves in scalar, vector, and 
multidimensional nonlinear systems, J. Phys. A: Math. Theor. 50, 463001 (2017).

[15] J. M. Soto-Crespo, N. Devine, and N. Akhmediev, Integrable Turbulence and Rogue Waves: Breathers or Solitons? Phys. Rev. Lett. 116, 103901 (2016).

[16] B. Kibler, A. Chabchoub, A. Gelash, N. Akhmediev, and V. E. Zakharov, Superregular Breathers in Optics and Hydrodynamics: Omnipresent Modulation Instability Beyond Simple Periodicity, Phys. Rev. X 5, 041026 (2015).

[17] D. Pierangeli, M. Flammini, L. Zhang, G. Marcucci, A. J. Agranat, P. G. Grinevich, P. M. Santini, C. Conti, and E. DelRe, Observation of Fermi-Pasta-Ulam-Tsingou Recurrence and Its Exact Dynamics, Phys. Rev. X 8, 041017 (2018).

[18] G. Vanderhaegen, C. Naveau, P. Szriftgiser, A. Kudlinski, M. Conforti, A. Mussot, M. Onorato, S. Trillo, A. Chabchoub, and N. Akhmediev, "Extraordinary" modulation instability in optics and hydrodynamics, Proc. Natl. Acad. Sci. USA 118, e2019348118 (2021).

[19] K. Dysthe, H. E. Krogstad, and P. Müller, Oceanic rogue waves, Annu. Rev. Fluid Mech. 40, 287 (2008).

[20] B. Kibler, J. Fatome, C. Finot, G. Millot, F. Dias, G. Genty, N. Akhmediev, and J. M. Dudley, The Peregrine soliton in nonlinear fibre optics, Nat. Phys. 6, 790 (2010).

[21] B. Frisquet, B. Kibler, P. Morin, F. Baronio, M. Conforti, G. Millot, and S. Wabnitz, Optical dark rogue wave, Sci. Rep. 6, 20785 (2016).

[22] F. Baronio, B. Frisquet, S. Chen, G. Millot, S. Wabnitz, and B. Kibler, Observation of a group of dark rogue waves in a telecommunication optical fiber, Phys. Rev. A 97, 013852 (2018).

[23] D. H. Peregrine, Water waves, nonlinear Schrödinger equations and their solutions, J. Aust. Math. Soc., Ser. B, Appl. Math. 25, 16 (1983).

[24] V. I. Shrira and V. V. Geogjaev, What makes the Peregrine soliton so special as a prototype of freak waves? J. Eng. Math. 67, 11 (2010).

[25] N. Akhmediev, A. Ankiewicz, and M. Taki, Waves that appear from nowhere and disappear without a trace, Phys. Lett. A 373, 675 (2009).

[26] W. Wang, L. Bu, D. Cheng, Y. Ye, S. Chen, and F. Baronio, Ultraslow Kuznetsov-Ma solitons and Akhmediev breathers in a cold three-state medium exposed to nanosecond optical pulses, OSA Continuum 4, 1488 (2021).

[27] A. Tikan, C. Billet, G. El, A. Tovbis, M. Bertola, T. Sylvestre, F. Gustave, S. Randoux, G. Genty, P. Suret, and J. M. Dudley, Universality of the Peregrine Soliton in the Focusing Dynamics of the Cubic Nonlinear Schrödinger Equation, Phys. Rev. Lett. 119, 033901 (2017).

[28] H. Bailung, S. K. Sharma, and Y. Nakamura, Observation of Peregrine Solitons in a Multicomponent Plasma with Negative Ions, Phys. Rev. Lett. 107, 255005 (2011).

[29] A. Chabchoub, Tracking Breather Dynamics in Irregular Sea State Conditions, Phys. Rev. Lett. 117, 144103 (2016).

[30] S. Chen and D. Mihalache, Vector rogue waves in the Manakov system: Diversity and compossibility, J. Phys. A: Math. Theor. 48, 215202 (2015).

[31] Y. Ye, Y. Zhou, S. Chen, F. Baronio, and P. Grelu, General rogue wave solutions of the coupled Fokas-Lenells equations and non-recursive Darboux transformation, Proc. R. Soc. A 475, 20180806 (2019).
[32] F. Baronio, M. Conforti, A. Degasperis, S. Lombardo, M. Onorato, and S. Wabnitz, Vector Rogue Waves and Baseband Modulation Instability in the Defocusing Regime, Phys. Rev. Lett. 113, 034101 (2014).

[33] S. Chen, J. M. Soto-Crespo, and P. Grelu, Coexisting rogue waves within the $(2+1)$-component long-wave-short-wave resonance, Phys. Rev. E 90, 033203 (2014).

[34] S. Chen, Y. Ye, J. M. Soto-Crespo, P. Grelu, and F Baronio, Peregrine Solitons Beyond the Threefold Limit and Their Two-Soliton Interactions, Phys. Rev. Lett. 121, 104101 (2018).

[35] S. Chen, C. Pan, P. Grelu, F. Baronio, and N. Akhmediev, Fundamental Peregrine Solitons of Ultrastrong Amplitude Enhancement through Self-Steepening in Vector Nonlinear Systems, Phys. Rev. Lett. 124, 113901 (2020).

[36] N. Akhmediev, A. Ankiewicz, and J. M. Soto-Crespo, Rogue waves and rational solutions of the nonlinear Schrödinger equation, Phys. Rev. E 80, 026601 (2009).

[37] Y. Ohta and J. Yang, General high-order rogue waves and their dynamics in the nonlinear Schrödinger equation, Proc. R. Soc. A 468, 1716 (2012).

[38] J. S. He, H. R. Zhang, L. H. Wang, K. Porsezian, and A. S. Fokas, Generating mechanism for higher-order rogue waves, Phys. Rev. E 87, 052914 (2013).

[39] A. Chabchoub and N. Akhmediev, Observation of rogue wave triplets in water waves, Phys. Lett. A 377, 2590 (2013).

[40] S. Chen, J. M. Soto-Crespo, and P. Grelu, Dark three-sister rogue waves in normally dispersive optical fibers with random birefringence, Opt. Express 22, 27632 (2014).

[41] A. Chabchoub, N. Hoffmann, M. Onorato, and N. Akhmediev, Super Rogue Waves: Observation of a Higher-Order Breather in Water Waves, Phys. Rev. X 2, 011015 (2012).

[42] S. Chen, Y. Zhou, L. Bu, F. Baronio, J. M. Soto-Crespo, and D. Mihalache, Super chirped rogue waves in optical fibers, Opt. Express 27, 11370 (2019).

[43] N. Akhmediev, J. M. Soto-Crespo, and A. Ankiewicz, How to excite a rogue wave, Phys. Rev. A 80, 043818 (2009).

[44] N. Akhmediev, J. M. Soto-Crespo, and A. Ankiewicz, Could rogue waves be used as efficient weapons against enemy ships? Eur. Phys. J.: Spec. Top. 185, 259 (2010).

[45] A. N. Pisarchik, R. Jaimes-Reátegui, R. Sevilla-Escoboza, G. Huerta-Cuellar, and M. Taki, Rogue Waves in a Multistable System, Phys. Rev. Lett. 107, 274101 (2011).

[46] A. E. Kaplan, Bistable Solitons, Phys. Rev. Lett. 55, 1291 (1985).

[47] C. Bao, W. Chang, C. Yang, N. Akhmediev, and S. T. Cundiff, Observation of Coexisting Dissipative Solitons in a ModeLocked Fiber Laser, Phys. Rev. Lett. 115, 253903 (2015).

[48] A. U. Nielsen, B. Garbin, S. Coen, S. G. Murdoch, and M. Erkintalo, Coexistence and Interactions between Nonlinear States with Different Polarizations in a Monochromatically Driven Passive Kerr Resonator, Phys. Rev. Lett. 123, 013902 (2019).

[49] J. Moses and F. W. Wise, Controllable Self-Steepening of U1trashort Pulses in Quadratic Nonlinear Media, Phys. Rev. Lett. 97, 073903 (2006).

[50] F. Baronio, C. De Angelis, M. Marangoni, C. Manzoni, R. Ramponi, and G. Cerullo, Spectral shift of femtosecond pulses in nonlinear quadratic PPSLT crystals, Opt. Express 14, 4774 (2006). 
[51] J. Moses, B. A. Malomed, and F. W. Wise, Self-steepening of ultrashort optical pulses without self-phase-modulation, Phys. Rev. A 76, 021802(R) (2007).

[52] H. N. Chan, K. W. Chow, D. J. Kedziora, R. H. J. Grimshaw, and E. Ding, Rogue wave modes for a derivative nonlinear Schrödinger model, Phys. Rev. E 89, 032914 (2014).

[53] J. Zhang, W. Liu, D. Qiu, Y. Zhang, K. Porsezian, and J. He, Rogue wave solutions of a higher-order Chen-Lee-Liu equation, Phys. Scr. 90, 055207 (2015).

[54] S. Chen, F. Baronio, J. M. Soto-Crespo, Y. Liu, and P. Grelu, Chirped Peregrine solitons in a class of cubic-quintic nonlinear Schrödinger equations, Phys. Rev. E 93, 062202 (2016).

[55] Y. Zhang, L. Guo, A. Chabchoub, and J. He, Higher-order rogue wave dynamics for a derivative nonlinear Schrödinger equation, Rom. J. Phys. 62, 102 (2017).

[56] L.-C. Zhao, L. Duan, P. Gao, and Z.-Y. Yang, Vector rogue waves on a double-plane wave background, Europhys. Lett. 125, 40003 (2019).

[57] Y. Ye, L. Bu, W. Wang, S. Chen, F. Baronio, and D. Mihalache, Peregrine solitons on a periodic background in the vector cubicquintic nonlinear Schrödinger equation, Front. Phys. 8, 596950 (2020).

[58] D. J. Kedziora, A. Ankiewicz, and N. Akhmediev, Rogue waves and solitons on a cnoidal background, Eur. Phys. J.: Spec. Top. 223, 43 (2014).

[59] G. Xu, A. Chabchoub, D. E. Pelinovsky, and B. Kibler, Observation of modulation instability and rogue breathers on stationary periodic waves, Phys. Rev. Research 2, 033528 (2020).

[60] J. Chen, D. E. Pelinovsky, and R. E. White, Rogue waves on the double-periodic background in the focusing nonlinear Schrödinger equation, Phys. Rev. E 100, 052219 (2019).

[61] J. Chen, D. E. Pelinovsky, and R. E. White, Periodic standing waves in the focusing nonlinear Schrödinger equation: Rogue waves and modulation instability, Physica D 405, 132378 (2020).

[62] B.-F. Feng, L. Ling, and D. A. Takahashi, Multi-breather and high-order rogue waves for the nonlinear Schrödinger equation on the elliptic function background, Stud. Appl. Math. 144, 46 (2020).
[63] J. Bernatska, V. Enolski, and A. Nakayashiki, Sato Grassmannian and degenerate sigma function, Commun. Math. Phys. 374, 627 (2020).

[64] Y. Kodama and A. Hasegawa, Nonlinear pulse propagation in a monomode dielectric guide, IEEE J. Quantum Electron. 23, 510 (1987).

[65] G. P. Agrawal, Nonlinear Fiber Optics, 4th ed. (Academic, San Diego, 2007).

[66] J. U. Kang, G. I. Stegeman, J. S. Aitchison, and N. Akhmediev, Observation of Manakov Spatial Solitons in AlGaAs Planar Waveguides, Phys. Rev. Lett. 76, 3699 (1996).

[67] M. Onorato, A. R. Osborne, and M. Serio, Modulational Instability in Crossing Sea States: A Possible Mechanism for the Formation of Freak Waves, Phys. Rev. Lett. 96, 014503 (2006).

[68] T. Tsuchida and M. Wadati, New integrable systems of derivative nonlinear Schrödinger equations with multiple components, Phys. Lett. A 257, 53 (1999).

[69] V. B. Matveev and M. A. Salle, Darboux Transformation and Solitons (Springer, Berlin, 1991).

[70] S. Chen and L.-Y. Song, Rogue waves in coupled Hirota systems, Phys. Rev. E 87, 032910 (2013).

[71] B. Guo, L. Ling, and Q. P. Liu, Nonlinear Schrödinger equation: Generalized Darboux transformation and rogue wave solutions, Phys. Rev. E 85, 026607 (2012).

[72] N. S. Ginzburg, R. M. Rozental, A. S. Sergeev, A. E. Fedotov, I. V. Zotova, and V. P. Tarakanov, Generation of Rogue Waves in Gyrotrons Operating in the Regime of Developed Turbulence, Phys. Rev. Lett. 119, 034801 (2017).

[73] R. W. Boyd, Nonlinear Optics, 3rd ed. (Academic, San Diego, 2008).

[74] A. V. Buryak and Y. S. Kivshar, Multistability of ThreeWave Parametric Self-Trapping, Phys. Rev. Lett. 78, 3286 (1997).

[75] B. A. Malomed, I. M. Skinner, P. L. Chu, and G. D. Peng, Symmetric and asymmetric solitons in twin-core nonlinear optical fibers, Phys. Rev. E 53, 4084 (1996).

[76] Y. Tanguy, T. Ackemann, W. J. Firth, and R. Jäger, Realization of a Semiconductor-Based Cavity Soliton Laser, Phys. Rev. Lett. 100, 013907 (2008). 